\title{
MINOR-MINIMAL PLANAR GRAPHS OF EVEN BRANCH-WIDTH
}

\author{
TORSTEN INKMANN AND ROBIN THOMAS
}

\begin{abstract}
Let $k \geq 1$ be an integer, and let $H$ be a graph with no isolated vertices embedded in the projective plane such that every homotopically non-trivial closed curve intersects $H$ at least $k$ times, and the deletion and contraction of any edge in this embedding results in an embedding that no longer has this property. Let $G$ be the planar double cover of $H$ obtained by lifting $G$ into the universal covering space of the projective plane, the sphere. We prove that $G$ is minor-minimal of branch-width $2 k$. We also exhibit examples of minor-minimal planar graphs of branch-width 6 that do not arise this way.
\end{abstract}

\section{INTRODUCTION}

The projective plane $\mathbb{P}$ is obtained from a closed disk by identifying diagonally opposite pairs of points on the boundary of the disk. Given a graph $H$ embedded in $\mathbb{P}$ its planar double cover $G$ is the lift of $H$ into the universal covering space of $\mathbb{P}$, the sphere. Thus to every vertex $v$ of $H$ there correspond two vertices $v_{1}, v_{2}$ of $G$; we say that $v$ is the projection of $v_{1}$ and $v_{2}$, and that $v_{1}$ and $v_{2}$ are the lifts of $v$. Similarly, we speak of projections and lifts of paths, cycles, walks, and faces. This construction is illustrated in Figure 1, where the Dodecahedron is shown to be a planar double cover of the Petersen graph. In particular, if $W$ is a walk in $G$ with ends the two lifts of a vertex $v \in V(H)$, then the projection of $W$ is a homotopically non-trivial closed walk in $H$ with both ends $v$.

Let $H$ and $G$ be as in the above paragraph. Our objective is to relate the representativity of $H$ (also known as face-width) and the branch-width of $G$, two important parameters that we now review. A graph is a minor of another if the first can be obtained from a subgraph of the second by contracting edges. Let $k \geq 0$ be an integer. We say that a graph $H$ embedded in $\mathbb{P}$ (a "projective plane graph") is $k$-representative if every homotopically non-trivial closed curve in $\mathbb{P}$ intersects $H$ at least $k$ times. (We mean all curves, including those passing through vertices of $H$; in fact, it suffices to restrict oneself to curves that intersect the graph only in vertices.) This concept has received a lot of attention in the literature; we refer to [7] for more information. We say that $H$ is minor-minimal $k$-representative if $H$ is $k$-representative, has no isolated vertices and for every edge $e$ of $H$ the embedded graphs obtained from $H$ by deleting and contracting $e$ are no longer $k$-representative. (One could also define a related concept where we say that no proper minor of $H$, taken as an abstract graph, has a $k$ representative embedding in $\mathbb{P}$. For $k \geq 3$ these two notions coincide, but we use the former definition.) Randby [8] proved that given two minor-minimal $k$-representative graphs in $\mathbb{P}$, each can be obtained from the other by means of repeated application of $\Delta Y$ - and $Y \Delta$ exchanges. Since for each $k$ there is a natural example of a minor-minimal $k$-representative projective plane graph, namely the $k \times k$ projective grid, Randby's result gives a convenient way to generate all minor-minimal $k$-representative projective plane graphs. Incidentally, Schrijver [12] proved an analogue of Randby's result for the torus, as well as a related result for arbitrary orientable surfaces [11.

A branch-decomposition of a graph $G$ is pair $(T, \eta)$, where $T$ is a tree with all vertices of degree one or three, and $\eta$ is a bijection between the leaves (vertices of degree one) of $T$ and

Partially supported by NSF grant DMS-0701077. 7 July 2010. 

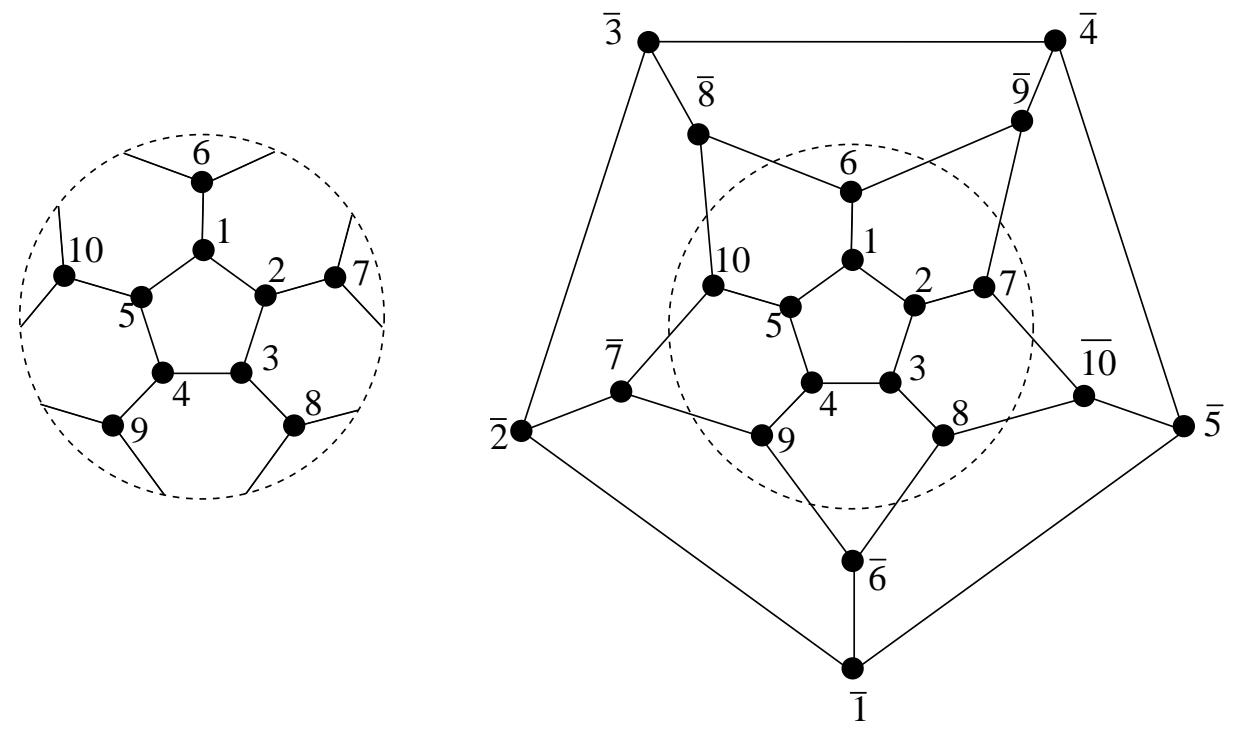

Figure 1. An embedding of the Petersen graph in $\mathbb{P}$ and its planar double cover, the Dodecahedron

$E(G)$. For $f \in E(T)$ let $T_{1}, T_{2}$ be the two components of $T \backslash f$, and let $X_{i}$ be the set of leaves of $T$ that belong to $T_{i}$. We define the order of $f$ to be the number of vertices of $G$ incident both with an edge in $\eta\left(X_{1}\right)$ and an edge in $\eta\left(X_{2}\right)$. The width of $(T, \eta)$ is the maximum order of an edge of $T$, and the branch-width of $G$ is the minimum width of a branch-decomposition of $G$, or 0 if $|E(G)| \leq 1$, in which case $G$ has no branch-decomposition.

Computing branch-width is NP-hard [13, but there is a polynomial time algorithm when $G$ is planar [13]. Thus one might expect that planar branch-width is better behaved in other respects as well, such as in terms of excluded minors. Since taking minors does not increase branch-width, as is easily seen, graphs of branch-width at most $t$ are characterized by excluded minors, the list of minor-minimal graphs of branch-width $t+1$. It follows from [9] that this list is finite.

Our first result is that planar double covers of minor-minimal $k$-representative projective plane graphs are excluded minors for odd branch-width:

Theorem 1.1. Let $k \geq 1$ be an integer, let $H$ be a minor-minimal $k$-representative projective plane graph, and let $G$ be the double cover of $H$. Then $G$ is a minor-minimal graph of branchwidth $2 k$.

Thus, in view of Randby's theorem mentioned above, this gives a description of a class of minor-minimal graphs of branch-width $2 k$. Since for $k=1$ and $k=2$ this class actually includes all minor-minimal graphs of branch-width $2 k$ [2, 3, 5], one might wonder whether this holds in general. Unfortunately, it does not. In Section 4 we exhibit examples of minor-minimal planar graphs of branch-width 6 that are not double covers of any graph.

The paper is organized as follows. We prove the lower bound for Theorem 1.1 in Section 2 and the corresponding upper bound in Section 3. The examples are presented in Section 4 .

The second author would like to acknowledge helpful conversations with P. D. Seymour from the summer of 1990; in particular, the question whether all minor-minimal graphs of even branch-width arise as in Theorem 1.1 was inspired by those conversations. The second author would also like to acknowledge that the results of this paper appeared (in a slightly different form) in the $\mathrm{PhD}$ dissertation [4] of the first author. 


\section{LOWER BOUND}

We will make use of the result of [13] that the branch-width of a planar graph $G$ is equal to one half the "carving-width" of the "medial graph" of $G$. Here are the definitions. Let $G$ be a graph drawn in a surface in such a way that every face of $G$ is homeomorphic to an open disk. Let us choose, for each vertex $v \in V(G)$, one of the two cyclic orderings of edges incident with $v$ (with each loop occurring twice in the ordering) and designate it as clockwise. By an angle at $v$ we mean an ordered pair $\left(e, e^{\prime}\right)$ of edges incident with $v$ such that $e^{\prime}$ immediately follows $e$ in the clockwise order around $v$. To each angle there naturally corresponds a face $f$ of $G$ incident with $e$ and $e^{\prime}$. The medial graph of $G$ is the graph $M$ defined as follows. For each edge $e \in E(G)$ choose a vertex $x_{e}$ positioned in the interior of $e$, and for each angle $\left(e, e^{\prime}\right)$ choose an edge joining $x_{e}$ and $x_{e^{\prime}}$ inside the face of $G$ that corresponds to the angle $\left(e, e^{\prime}\right)$ in a small neighborhood of $e$ and $e^{\prime}$. There is a certain ambiguity when the same face corresponds to different angles, but there is a natural interpretation under which the medial graph is unique up to homotopic shifts of edges. Each face of $M$ corresponds to either a unique vertex of $G$ or a unique face of $G$, and if $G$ has no loops or cut edges, then every face of $M$ is bounded by a cycle.

A carving in a graph $G$ is a pair $(T, \eta)$, where $T$ is a tree with all vertices of degree one or three, and $\eta$ is a bijection from the leaves of $T$ to $V(G)$. Similarly as in a branchdecomposition, each edge $f$ of $T$ determines a cut in $G$. The width of $(T, \eta)$ is the maximum order of those cuts, over all $f \in E(T)$. The carving-width of $G$ is the minimum width of a carving in $G$. The following is [13, Theorem 7.2].

Theorem 2.1. Let $G$ be a connected plane graph with at least two edges, and let $M$ be its medial graph. Then the branch-width of $G$ is half the carving-width of $M$.

Thus it suffices to bound the carving-width of the medial graph of $G$ from Theorem 1.1 . We will use the concept of an "antipodality," introduced in [13. Let $G$ be a connected plane graph with planar dual $G^{*}$, let $F(G)$ be the faces of $G$, and let $k \geq 0$ be an integer. An antipodality in $G$ of range $\geq k$ is a function $\alpha$ with domain $E(G) \cup F(G)$, such that for all $e \in E(G), \alpha(e)$ is a subgraph of $G$ with at least one vertex and for all $f \in F(G), \alpha(f)$ is a non-empty subset of $V(G)$, satisfying:

(A1) If $e \in E(G)$, then no end of $e$ belongs to $V(\alpha(e))$

(A2) If $e \in E(G), f \in F(G)$, and $e$ is incident with $f$, then $\alpha(f) \subseteq V(\alpha(e))$, and every component of $\alpha(e)$ has a vertex in $\alpha(f)$

(A3) If $e_{1} \in E(G)$ and $e_{2} \in E\left(\alpha\left(e_{1}\right)\right)$ then every closed walk of $G^{*}$ using $e_{1}^{*}$ and $e_{2}^{*}$ has length $\geq k$.

The following is a special case of [13, Theorem 4.1]; we will only need the easier "if" part.

Theorem 2.2. Let $M$ be a connected plane graph on at least two vertices, and let $k \geq 0$ be an integer. Then $M$ has carving-width at least $k$ if and only if either some vertex of $M$ has degree at least $k$, or $M$ has an antipodality of range $\geq k$.

As we will see at the end of the next section, the following lemma and Theorems 2.1 and 2.2 imply that the planar double cover of a minor-minimal $k$-representative projective plane graph has branch-width at least $2 k$.

Lemma 2.3. Let $k \geq 2$ be an integer, let $H$ be a $k$-representative projective plane graph, let $G$ be a planar double cover of $H$, and let $M$ be the medial graph of $G$. Then $M$ has an antipodality of range $\geq 4 k$.

Proof. First we notice that since $k \geq 2$ we may assume (by considering a subgraph of $H$ ) that the graph $G$ has no loops or cut edges. For $v \in V(G)$ and a face $f \in F(G)$ incident 
with $v$ there is a unique edge $a_{v, f}$ of $M$ incident with $v^{*}$ and $f^{*}$, where $v^{*}$ and $f^{*}$ denote the faces of $M$ corresponding to $v$ and $f$, respectively. For $v \in V(G)$ let $v^{\prime} \in V(G)$ be the other vertex of $G$ with the same projection as $v$, and for $f \in F(G)$ let $f^{\prime}$ be defined analogously. Finally, for $x \in V(G) \cup F(G)$ let $C_{x}$ denote the cycle bounding the face of $M$ corresponding to $x$. We note that $C_{x}$ is indeed a cycle, because $G$ has no loops or cut edges. We define the function $\alpha$ as follows:

$$
\begin{aligned}
\alpha\left(a_{v, f}\right) & =C_{v^{\prime}} \cup C_{f^{\prime}} \text { for every } v \in V(G) \text { and } f \in F(G) \\
\alpha(f) & =V\left(C_{f^{\prime}}\right) \text { for every } f \in F(M)
\end{aligned}
$$

We claim that $\alpha$ defines an antipodality of range $4 k$ in $M$. Conditions (A1) and (A2) follow easily from the fact that $G$ has no loops or cut edges and that each $\alpha\left(a_{v, f}\right)$ is connected.

To prove (A3) suppose for a contradiction that there is a closed walk $W$ in $M^{*}$ of length at most $4 k-1$ that uses $a^{*}$ and $b^{*}$ for some $a=a_{v, f} \in E(M)$ and $b \in E\left(\alpha\left(a_{v, f}\right)\right)$. Thus $W$ uses both $v^{*}$ and $f^{*}$, and at least one of $\left(v^{\prime}\right)^{*},\left(f^{\prime}\right)^{*}$. In either case, $W$ includes a subwalk $W^{\prime}$ of length at most $2 k-1$ from $x^{*}$ to $\left(x^{\prime}\right)^{*}$ for some $x \in V(G) \cup F(G)$. Now the projection of $W^{\prime}$ is a homotopically non-trivial closed walk of length at most $2 k-1$ in the dual of the medial graph of $H$. That, in turn, implies that $H$ is not $k$-representative, a contradiction. Hence $\alpha$ is an antipodality, as desired.

\section{UPPER BOUND}

In this section we show that if $G$ is as in Theorem 1.1, then every proper minor of $G$ has branch-width at most $2 k-1$. Again, we find it convenient to work with carving-width of the medial graph. Thus we need to translate minimal $k$-representativity into the language of medial graphs. Let $G$ be a graph drawn in a surface, let $v$ be a vertex of $G$ of degree four, let $e_{1}, e_{2}, e_{3}, e_{4}$ be the four edges of $G$ incident with $v$ listed in the cyclic order of appearance around $v$, let $v_{i}$ be the other end of $e_{i}$, and let $f_{i}$ be the face of $G$ incident with $e_{i}$ and $e_{i-1}$, where $e_{0}$ means $e_{4}$. Let $G^{\prime}$ be obtained from $G \backslash v$ by inserting two edges, one with ends $v_{1}$ and $v_{4}$ in the face $f_{1}$ and the other with ends $v_{2}$ and $v_{3}$ in the face $f_{3}$. We say that $G^{\prime}$ was obtained from $G$ by opening at $v$ through the faces $f_{2}$ and $f_{4}$, or simply by opening at $v$. Let $k \geq 1$ be an integer. A 4-regular graph $G$ drawn in $\mathbb{P}$ is $k$-tight if every homotopically non-trivial closed walk in $G^{*}$ has length at least $k$, and for every graph $J$ obtained from $G$ by opening at some vertex there exists a homotopically non-trivial closed walk in $J^{*}$ of length at most $k-1$. We say that $G$ is tight if it is $k$-tight for some integer $k \geq 1$. The following is shown in [11] and is also easy to see.

Lemma 3.1. Let $k \geq 1$ be an integer, let $G$ be a connected graph in the projective plane, and let $M$ be the medial graph of $G$. Then $G$ is a minor-minimal k-representative graph in $\mathbb{P}$ if and only if $M$ is $2 k$-tight.

We will need the following characterization of tight graphs in terms of straight ahead decompositions. Let $G$ be a graph with all vertices of degree four or one drawn in a surface. Let $v$ be a vertex of $G$ of degree four, and let $e_{1}, e_{2}, e_{3}, e_{4}$ be the edges of $G$ incident with $v$ listed in the order in which they appear around $v$. We say that the edges $e_{1}$ and $e_{3}$ are opposite. Let $F_{1}, F_{2}, \ldots, F_{r}$ be the equivalence classes of the transitive closure of the opposite relation, and let $G_{i}$ be the subgraph of $G$ with edge-set $F_{i}$ and vertex-set all vertices incident with edges of $F_{i}$. We say that $G_{1}, G_{2}, \ldots, G_{r}$ is the straight ahead decomposition of $G$. The next result follows from a theorem of Lins [6]. Schrijver [10] obtained an analogous result for orientable surfaces. 
Theorem 3.2. Let $G$ be a 4-regular graph drawn in the projective plane. Then $G$ is tight if and only if the straight ahead decomposition of $G$ consists of homotopically non-trivial cycles such that every two intersect exactly once.

As usual, a walk in a graph $G$ is an alternating sequence of vertices and edges of $G$. It has an origin and terminus, called its ends. A walk is closed if its ends are equal. Even though any vertex of a closed walk may be regarded as its origin and terminus, for our purposes changing the ends results in a different walk. This subtle point will be important later.

Let $G$ be a graph drawn in a surface, let $f, f^{\prime}$ be two faces of $G$, and let $W_{1}, W_{2}$ be two walks in $G^{*}$ with origin $f^{*}$ and terminus $\left(f^{\prime}\right)^{*}$. Let $v$ be a vertex of $G$ of degree four, let $e_{1}, e_{2}, e_{3}, e_{4}$ be the four edges of $G$ incident with $v$ listed in the cyclic order of appearance around $v$, and let $f_{i}$ be the face of $G$ incident with $e_{i}$ and $e_{i-1}$, where $e_{0}$ means $e_{4}$. If $W_{1}$ includes the subwalk $f_{1}^{*}, e_{1}^{*}, f_{2}^{*}, e_{2}^{*}, f_{3}^{*}$, and $W_{2}$ is obtained from $W_{1}$ by replacing (one occurence of) that subwalk by the walk $f_{1}^{*}, e_{4}^{*}, f_{4}^{*}, e_{3}^{*}, f_{3}^{*}$, then we say that $W_{2}$ was obtained from $W_{1}$ by a $\Delta \nabla$-exchange. We write $W_{1}=W_{2} * v$; then also $W_{2}=W_{1} * v$. Let us remark the obvious fact that $W_{1}$ and $W_{2}$ have the same origin and terminus.

Let $\Delta$ denote the closed unit disk in $\mathbb{R}^{2}$. Let $G$ be a graph drawn in $\Delta$ such that the vertices $v_{1}, v_{2}, \ldots, v_{2 k}$ and only these vertices are drawn on the boundary of $\Delta$ in the order listed, where $v_{1}, v_{2}, \ldots, v_{2 k}$ have degree one and all other vertices of $G$ have degree four. Assume further that the straight ahead decomposition of $G$ is of the form $P_{1}, P_{2}, \ldots, P_{k}$, where each $P_{i}$ is a path with one end in $\left\{v_{1}, v_{2}, \ldots, v_{k}\right\}$ and the other end in $\left\{v_{k+1}, v_{k+2}, \ldots, v_{2 k}\right\}$. Finally, assume that for $i \neq j$ the paths $P_{i}$ and $P_{j}$ intersect in at most one vertex. In those circumstances we say that $G$ is a graft, and that $v_{1}, v_{2}, \ldots, v_{2 k}$ are its attachments. For $i=1,2, \ldots, 2 k$ let $e_{i}$ denote the unique edge of $G$ incident with $v_{i}$, and let $f_{i}$ be the face of $G$ incident with $e_{i-1}$ and $e_{i}$, where $e_{0}$ means $e_{2 k}$. Let us emphasize that $G$ is embedded in $\Delta$, and hence $f_{i}$ and $f_{i+1}$ are distinct faces, each incident with a segment of the boundary of $\Delta$. Let $G^{*}$ denote the geometric dual of $G$. By a broom in $G$ we mean a walk in $G^{*}$ from $f_{1}^{*}$ to $f_{k+1}^{*}$ of length exactly $k$. (The existence of $P_{1}, P_{2}, \ldots, P_{k}$ implies that every walk in $G^{*}$ from $f_{1}^{*}$ to $f_{k+1}^{*}$ has length at least $k$.) There are two natural examples of brooms, namely the walks with edge-sets $f_{1}^{*}, f_{2}^{*}, \ldots, f_{k+1}^{*}$ and $f_{1}^{*}, f_{2 k}^{*}, f_{2 k-1}^{*}, \ldots, f_{k+1}^{*}$. Those two brooms will be called the extreme brooms of $G$.

Let $G$ be a graph drawn in a surface with every vertex of degree four or one, let $f, f^{\prime}$ be two faces of $G$, and let $W_{0}$ and $W$ be two walks in $G^{*}$ with origin $f$ and terminus $f^{\prime}$. We say that $G$ is sweepable from $W_{0}$ to $W$ if the vertices of $G$ of degree four can be numbered $v_{1}, v_{2}, \ldots, v_{n}$ so that $W_{i}:=W_{i-1} * v_{i}$ is well-defined for all $i=1,2, \ldots, n$ and $W_{n}=W$.

Lemma 3.3. Let $k \geq 1$ be an integer, let $G$ be a graft with attachments $v_{1}, v_{2}, \ldots, v_{2 k}$, and let $W, W^{\prime}$ be the extreme brooms of $G$. Then $G$ is sweepable from $W$ to $W^{\prime}$.

Proof. Let $P_{1}, P_{2}, \ldots, P_{k}$ be the straight ahead decomposition of $G$, numbered so that $v_{i}$ is an end of $P_{i}$ for $i=1,2, \ldots, k$. If the paths $P_{1}, P_{2}, \ldots, P_{k}$ are pairwise disjoint, then $G$ is a matching, and hence $W=W^{\prime}$ and the theorem holds. Thus we may assume that some two of the paths $P_{i}$ intersect, and we proceed by induction on $|V(G)|$. By a wedge we mean an ordered pair $(i, j)$ of distinct integers from $\{1,2, \ldots, k\}$ such that the paths $P_{i}$ and $P_{j}$ intersect in a (unique) vertex $v$, and $v_{i} P_{i} v$, the subpath of $P_{i}$ with ends $v_{i}$ and $v$, is not intersected by any other $P_{j^{\prime}}$. Since some two paths $P_{i}$ intersect, there exists a wedge, and so we may select a wedge $(i, j)$ with $|i-j|$ minimum.

We claim that $|i-j|=1$. To prove this claim we may assume that $i+1<j$. By planarity the path $P_{i+1}$ intersects $P_{i} \cup P_{j}$, and hence there exists a wedge $\left(i+1, j^{\prime}\right)$. We deduce from the minimality of $|i-j|$ that $j^{\prime} \notin\{i, i+1, \ldots, j\}$, and hence $P_{j^{\prime}}$ intersects $v_{i} P_{i} v \cup v P_{j} v_{j}$ by planarity. In fact, it intersects $v_{i} P_{i} v \cup v P_{j} v_{j}$ an even number of times. But $P_{j^{\prime}} \operatorname{does}$ not 
intersect $v_{i} P_{i} v$, because $(i, j)$ is a wedge, and hence $P_{j^{\prime}}$ intersects $P_{j}$ at least twice, contrary to the definiton of a graft. This contradiction proves our claim that $|i-j|=1$. It follows that both $v_{i} P_{i} v$ and $v_{j} P_{j} v$ have length one.

Thus we can apply a $\Delta \nabla$-exchange at the vertex $v$ to one of the brooms $W, W^{\prime}$, say to $W^{\prime}$, to obtain a broom $W^{\prime \prime}:=W^{\prime} * v$. Let $x_{i}$ be the neighbor of $v$ on $P_{i}$ other than $v_{i}$, and let $x_{j}$ be defined analogously. Let $G^{\prime}$ be obtained from $G$ by deleting $v, v_{i}, v_{j}$ and adding two new vertices of degree one, joined to $x_{i}$ and $x_{j}$, respectively. Then $G^{\prime}$ with its natural drawing in a disk forms a graft, and $W$ and $W^{\prime \prime}$ can be regarded as the two extreme brooms in $G^{\prime}$. By the induction hypothesis the graft $G^{\prime}$ is sweepable from $W$ to $W^{\prime \prime}$; by considering the corresponding ordering of vertices of $G^{\prime}$ and appending $v$ at the end we obtain a desired ordering of the vertices of $G$, showing that $G$ is sweepable from $W$ to $W^{\prime}$.

Theorem 3.4. Let $k \geq 1$ be an integer, let $H$ be a 4-regular $k$-tight graph in $\mathbb{P}$, and let $W$ be a homotopically non-trivial closed walk in $H^{*}$ of length $k$. Then $H$ is sweepable from $W$ to $W$.

Proof. By Theorem 3.2 the straight ahead decomposition of $H$ consists of $k$ homotopically non-trivial cycles such that every two of them intersect exactly once. We cut $H$ open along $W$ and construct a graft $G$ as follows. For every edge $e^{*}$ of $W$ we cut the corresponding edge $e$ into two by inserting two new vertices of degree one "in the middle of" $e$. The theorem follows by applying Theorem 3.3 to the resulting graft $G$.

Lemma 3.5. Let $k \geq 1$ be an integer, let $H$ be a 4 -regular $k$-tight graph in $\mathbb{P}$, and let $G$ be the planar double cover of $H$. Let $f_{1}, f_{2}$ be the two lifts of some face $f$ of $H$, and let $W_{1}$ be a walk of length $k$ in $G^{*}$ with origin $f_{1}$ and terminus $f_{2}$. Then $G$ is sweepable from $W_{1}$ to $W_{1}$.

Proof. Let $W$ be the projection of $W_{1}$, and let $W_{2}$ be the other lift of $W$. Then $W$ is a homotopically non-trivial closed walk in $H^{*}$. By Theorem $3.4 H$ is sweepable from $W$ to $W$; let $v_{1}, v_{2}, \ldots, v_{n}$ be the corresponding ordering of the vertices of $H$. Then each $v_{i}$ has a lift $v_{i}^{1} \in V(G)$ such that $W_{2}=W_{1} * v_{1}^{1} * v_{2}^{1} * \cdots * v_{n}^{1}$. Now let $v_{i}^{2}$ be the other lift of $v_{i}$. It follows that $W_{1}=W_{2} * v_{1}^{2} * v_{2}^{2} * \cdots * v_{n}^{2}$, and hence the sequence $v_{1}^{1}, v_{2}^{1}, \ldots, v_{n}^{1}, v_{1}^{2}, v_{2}^{2}, \ldots, v_{n}^{2}$ shows that $G$ is sweepable from $W_{1}$ to $W_{2}$.

Lemma 3.6. Let $k \geq 1$ be an integer, let $H$ be a 4 -regular $k$-tight graph in $\mathbb{P}$, and let $G$ be the planar double cover of $H$. Let $G_{1}$ be obtained from $G$ by opening at $\bar{u} \in V(G)$. Then $G_{1}$ has carving-width at most $2 k-1$.

Proof. Let $\bar{e}_{1}, \bar{e}_{2}, \bar{e}_{3}, \bar{e}_{4}$ be the edges of $G$ incident with $\bar{u}$ listed in cyclic order around $\bar{u}$, and let $\overline{f_{i}}$ be the face of $G$ incident with $\bar{e}_{i}$ and $\bar{e}_{i-1}$, where $\bar{e}_{0}$ means $\bar{e}_{4}$. Let the numbering be such that $G_{1}$ is obtained by opening at $\bar{u}$ through the faces $\overline{f_{2}}$ and $\overline{f_{4}}$. Let $u, e_{i}, f_{i}$ be the projections of $\bar{u}, \bar{e}_{i}, \overline{f_{i}}$, respectively. Let $H_{1}$ be the graph obtained from $H$ by opening at $u$ through the faces $f_{2}$ and $f_{4}$, and let $f_{24}$ be the resulting new face. Since $H$ is $k$-tight there exists a homotopically non-trivial closed walk $Z_{1}$ in $H_{1}^{*}$ of length $k-1$ with origin and terminus $f_{24}^{*}$. Since $H$ is $k$-tight, we deduce that $f_{24}^{*}$ is the only repeated vertex in $Z_{1}$. To $Z_{1}$ there corresponds a closed walk $Z$ in $H^{*}$ starting with $f_{2}^{*} e_{2}^{*} f_{3}^{*} e_{3}^{*} f_{4}^{*}$ and ending in $f_{2}^{*}$. Let $\bar{Z}$ be the lift of $Z$ that starts with $\left(\overline{f_{2}}\right)^{*}\left(\bar{e}_{2}\right)^{*}\left(\overline{f_{3}}\right)^{*}\left(\bar{e}_{3}\right)^{*}\left(\overline{f_{4}}\right)^{*}$ and ends in $\left(\tilde{f}_{2}\right)^{*}$, where $\tilde{f}_{2}$ is the other lift of $f_{2}$. There is a corresponding walk $\bar{Z}_{1}$ in $G_{1}^{*}$ with origin $\left(\bar{f}_{24}\right)^{*}$ and terminus $\left(\tilde{f}_{2}\right)^{*}$, where $\bar{f}_{24}$ is the face resulting from the opening of $G$ at $\bar{u}$ that creates $G_{1}$. 
By Lemma 3.5 the graph $G$ is sweepable from $\bar{Z}$ to $\bar{Z}$; let $v_{1}, v_{2}, \ldots, v_{n}$ be the corresponding ordering of the vertices of $G$, and let $W_{0}:=\bar{Z}$ and $W_{i}:=W_{i-1} * v_{i}$ be the corresponding walks. Since each $W_{i}$ starts at $\left(\overline{f_{2}}\right)^{*}$ we deduce that the $\Delta \nabla$-exchange at $\bar{u}$ replaces $\left(\overline{f_{2}}\right)^{*}\left(\bar{e}_{1}\right)^{*}\left(\overline{f_{1}}\right) *\left(\bar{e}_{4}\right)^{*}\left(\overline{f_{4}}\right)^{*}$ by $\left(\overline{f_{2}}\right) *\left(\bar{e}_{2}\right) *\left(\overline{f_{3}}\right) *\left(\bar{e}_{3}\right)^{*}\left(\overline{f_{4}}\right)^{*}$ or vice versa. From the symmetry we may assume that it replaces the former by the latter; then we may further assume that $\bar{u}=v_{n}$. We deduce that for all $i=1,2, \ldots, n-2$ the concatenation of $\bar{Z}_{1}$ and $W_{i}$ separates $\left\{v_{1}, v_{2}, \ldots, v_{i}\right\}$ from $\left\{v_{i+1}, v_{i+2}, \ldots, v_{n-1}\right\}$ in $G_{1}$. Now let $T$ be the tree obtained from a path with vertices $r_{1}, r_{2}, \ldots, r_{n-1}$ in order by adding, for each $i=2,3, \ldots, n-2$, a new vertex $t_{i}$ and joining it by an edge to $r_{i}$. Let $t_{1}=r_{1}$ and $t_{n-1}=r_{n-1}$, and let $\eta\left(t_{i}\right)=v_{i}$. Since $\bar{Z}_{1}$ has length $k-1$ and each $W_{i}$ has length $k$ we deduce that $(T, \eta)$ is a carving decomposition of $G_{1}$ of width at most $2 k-1$, as desired.

Proof of Theorem 1.1. Let $k \geq 1$ be an integer, let $H$ be a minor-minimal $k$-representative graph in $\mathbb{P}$, and let $G$ be the double cover of $H$. If $k=1$, then $H$ consists of one vertex and one edge, and hence $G$ has two vertices and two edges between them. It follows that the theorem holds in that case, and hence we may assume that $k \geq 2$.

We first show that $G$ has branch width at least $2 k$. Let $M$ be the medial graph of $G$. By Lemma 2.3 the graph $M$ has an antipodality of range $\geq 4 k$, and hence has carving-width at least $4 k$ by Theorem 2.2 . It follows from Theorem 2.1 that $G$ has branch-width at least $2 k$, as desired.

Let $G_{1}$ be obtained from $G$ by deleting or contracting an edge $e$. To complete the proof we show that $G_{1}$ has branch-width at most $2 k-1$; that will imply that the branch-width of $G$ is exactly $2 k$ and that it is minor-minimal. To this end let $M_{1}$ be the medial graph of $G_{1}$; then $M_{1}$ is obtained from $M$ by opening at the vertex of $M$ that correponds to $e$. Let $N$ be the medial graph of $H$; then $N$ is $2 k$-tight by Lemma 3.1 and $M$ is the planar double cover of $N$. By Lemma 3.6 the graph $M_{1}$ has carving-width at most $4 k-2$, and hence $G_{1}$ has branch-width at most $2 k-1$ by Theorem 2.1 , as desired.

\section{MinOR-MINIMAL PLANAR GRAPHS WHICH ARE NOT DOUBLE COVERS}

In [1] and [14] the authors found all the seven minor-minimal 3-representative embeddings of graphs in the projective plane. (There is a possible confusion here: these are embeddings of six different graphs, because one of those graphs has two different 3-representative embeddings in the projective plane. The six graphs are all members of the so-called Petersen family. The seventh member of the family, the graph obtained from $K_{4,4}$ by deleting one edge, does not embed in the projective plane.) By Theorem 1.1 the seven minor-minimal 3representative projective planar embeddings give rise to seven non-isomorphic minor-minimal planar graphs of branch-width 6. However, Figure 2 shows four additional minor-minimal planar graphs of branch-width 6, and their geometric duals provide four additional examples. In fact, none of these graphs is a double cover of any graph, because none of them has a fixed point-free automorphism of order two. We have generated those graphs using a computer program, which used as a subroutine an implementation of the algorithm from [13] written by Hicks. We are indebted to Ilya Hicks for letting us use his program.

Let $k \geq 1$ be an integer, and let $H, G$ be as in Theorem 1.1. Let $v \in V(H)$ have degree three, and let $H^{\prime}$ be obtained from $H$ by a $Y \Delta$-exchange at $v$; that is, $H^{\prime}$ is obtained from $H$ by deleting $v$ and adding an edge joining every pair of neighbors of $v$. The graph $H^{\prime}$ gives rise to a minor-minimal graph $G^{\prime}$ of branch-width $2 k$ as in Theorem 1.1, where $G^{\prime}$ can be regarded as having been obtained from $G$ by a $Y \Delta$-exchange at both the lifts of $v$, say $v_{1}$ and 

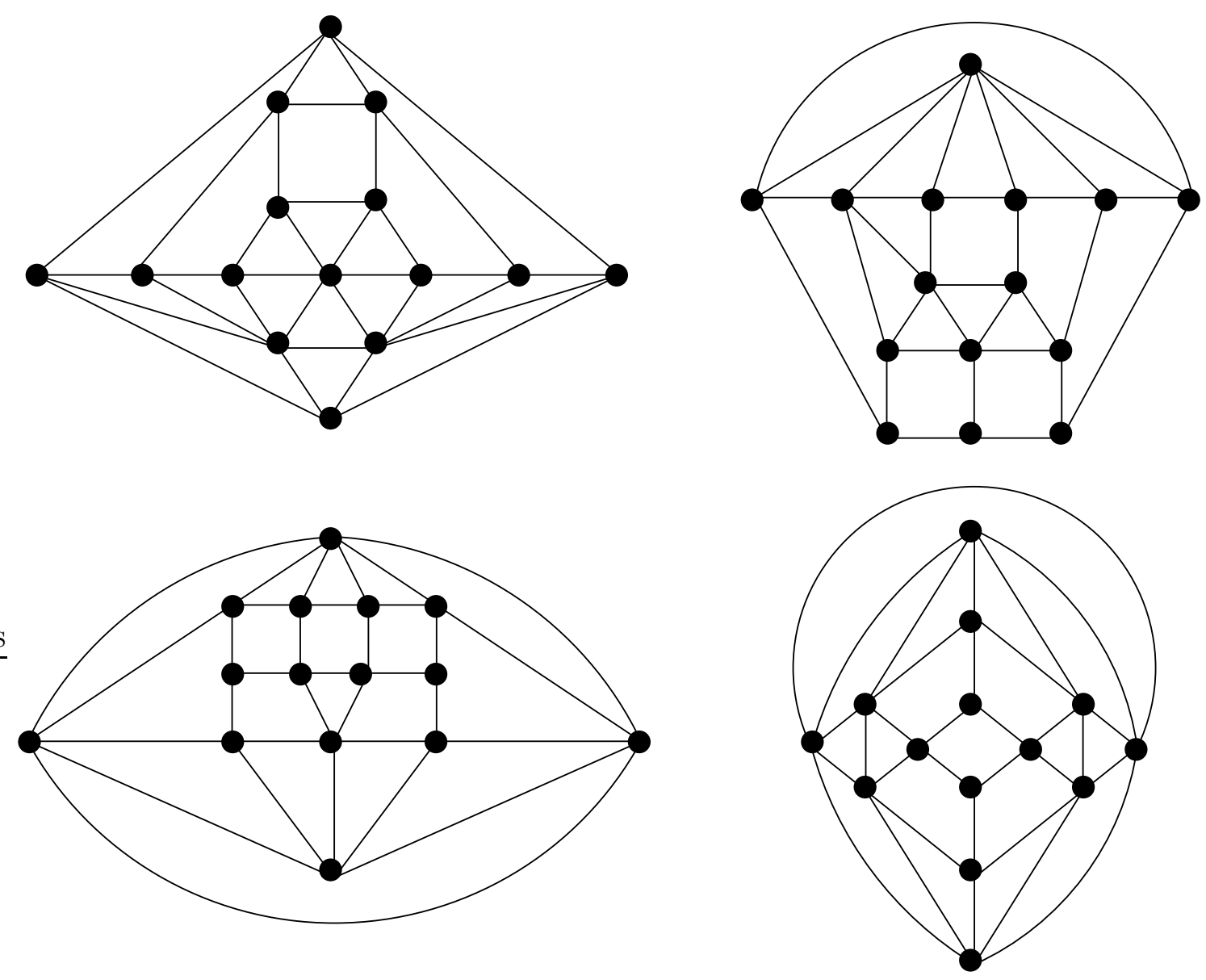

FiguRE 2. Minor-minimal graphs of branch-width 6 which are not double covers

$v_{2}$. Now a question arises about the status of the graph $G_{1}$ obtained from $G$ by performing a $Y \Delta$-exchange at $v_{1}$ only. For instance, when $k=2$ the graph $G$ could be the cube, but in that case the graph $G_{1}$ has branch-width 3 . We suspect that in general the graph $G_{1}$ has branch-width $2 k-1$, and that it can be shown using similar method as our proof of Theorem 1.1. However, it does not seem to follow easily from anything we have done, and so we do not pursue this question further.

Finally, here is an example of a minor-minimal planar graph of odd branch-width. For every $k \geq 2$ consider the planar $k \times(2 k+1)$ circular grid with $k$ concentric cycles and $2 k+1$ paths joining the cycles, and add a new vertex that is connected to all $2 k+1$ vertices on the innermost cycle to obtain a graph $G_{k}$. The graph $G_{3}$ is depicted in Figure 3, It can be shown that $G_{k}$ has branch-width exactly $2 k+1$ and is minor-minimal with that property.

\section{REFERENCES}

[1] D. Barnette, The minimal projective plane polyhedral maps, Applied geometry and discrete mathematics, 63-70, DIMACS Ser. Discrete Math. Theoret. Comput. Sci., 4, Amer. Math. Soc., Providence, RI, 1991.

[2] H. L. Bodlaender and D. M. Thilikos, Graphs with branchwidth at most three, J. Algorithms, 32 (1999), $167-194$.

[3] J. Dharmatilake, Binary Matroids With Branch-Width Three, PhD thesis, Ohio State University, 1994.

[4] T. Inkmann, Tree-based decompositions of graphs on surfaces and applications to the Traveling Salesman Problem, Ph.D. Dissertation, Georgia Institute of Technology, April 2008.

[5] E. Johnson and N. Robertson, private communication. 


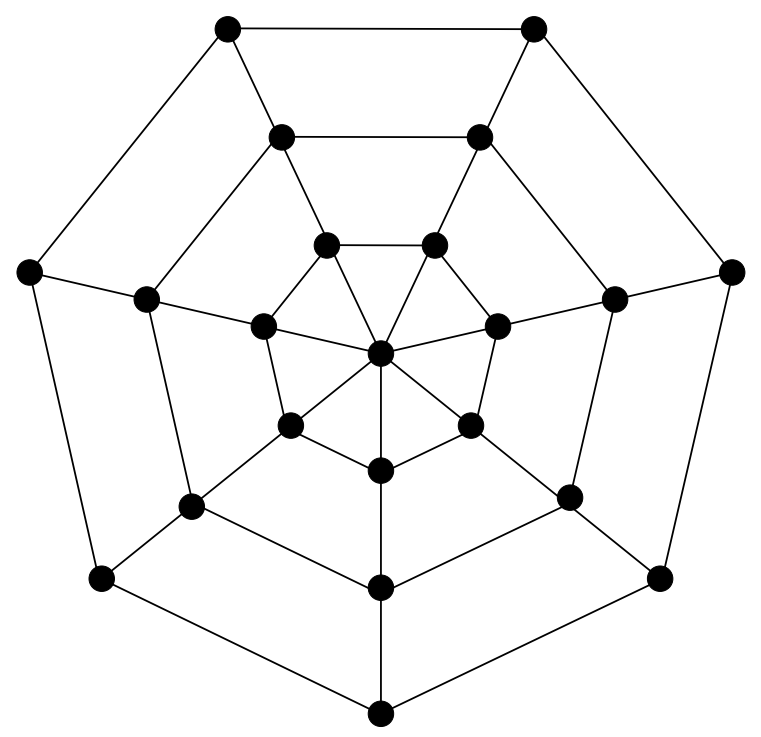

Figure 3. The graph $G_{3}$

[6] S. Lins, A minimax theorem on circuits in projective graphs, J. Combin. Theory Ser. B 30 (1981), $253-262$.

[7] B. Mohar and C. Thomassen, Graphs on surfaces, Johns Hopkins University Press, Baltimore, MD, 2001.

[8] S. P. Randby, Minimal embeddings in the projective plane, J. Graph Theory 25 (1997), 153-164.

[9] N. Robertson and P. D. Seymour, Graph Minors IV. Tree-width and well-quasi-ordering, J. Combin. Theory Ser. B 48 (1990), 227-254

[10] A. Schrijver, Decomposition of graphs on surfaces and a homotopic circulation theorem, J. Combin. Theory Ser. B 51 (1991), 161-210.

[11] A. Schrijver, On the uniqueness of kernels, J. Combin. Theory Ser. B 55 (1992), 146-160.

[12] A. Schrijver, Classification of minimal graphs of given representativity on the torus, J. Combin. Theory Ser. B 61 (1994), 217-236.

[13] P. D. Seymour and R. Thomas, Call routing and the ratcatcher, Combinatorica 14 (1994), 217-241.

[14] R. P. Vitray, Representativity and flexibility on the projective plane, Graph structure theory (Seattle, WA, 1991), 341-347, Contemp. Math., 147, Amer. Math. Soc., Providence, RI, 1993.

School of Mathematics, Georgia Institute of Technology, Atlanta, GA 30332-0160, USA 